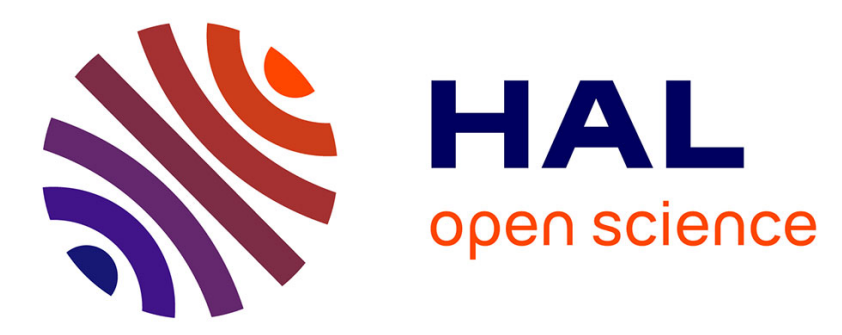

\title{
Modeling of Magneto-Mechanical Coupling Using Magnetic Volume Integral and Mechanical Finite-Element Methods
}

Anthony Carpentier, Nicolas Galopin, Olivier Chadebec, Gérard Meunier

\section{To cite this version:}

Anthony Carpentier, Nicolas Galopin, Olivier Chadebec, Gérard Meunier. Modeling of MagnetoMechanical Coupling Using Magnetic Volume Integral and Mechanical Finite-Element Methods. IEEE Transactions on Magnetics, 2014, 50 (2), 10.1109/TMAG.2013.2283600 . hal-02277364

\section{HAL Id: hal-02277364 \\ https://hal.science/hal-02277364}

Submitted on 30 Nov 2020

HAL is a multi-disciplinary open access archive for the deposit and dissemination of scientific research documents, whether they are published or not. The documents may come from teaching and research institutions in France or abroad, or from public or private research centers.
L'archive ouverte pluridisciplinaire $\mathbf{H A L}$, est destinée au dépôt et à la diffusion de documents scientifiques de niveau recherche, publiés ou non, émanant des établissements d'enseignement et de recherche français ou étrangers, des laboratoires publics ou privés. 


\title{
Modeling of Magneto-Mechanical Coupling Using Magnetic Volume Integral and Mechanical Finite-Element Methods
}

\author{
Anthony Carpentier ${ }^{1}$, Nicolas Galopin ${ }^{1,2}$, Olivier Chadebec ${ }^{1,3,4}$, and Gérard Meunier ${ }^{1,3}$ \\ ${ }^{1}$ Université de Grenoble-Alpes, G2Elab, Grenoble 38000, France \\ ${ }^{2}$ Université Joseph Fourier, G2Elab, Grenoble 38000, France \\ ${ }^{3}$ CNRS, G2Elab, Grenoble 38042, France \\ ${ }^{4}$ GRUCAD/EEL/CTC/UFSC, Florianópolis 88049, Brazil
}

\begin{abstract}
The magneto-mechanical coupling with deformable bodies is studied using different numerical methods for both physics. The magnetic behavior is considered linear and without magnetostrictive effects; then equations of the magneto-mechanical coupling can be solved separately. A volume integral method using the magnetic scalar potential formulation is used. This method is particularly well suited to compute fields without meshing the air domain. A local application of the virtual work principle, adapted to the integral formulation, is used to compute the nodal magnetic force distribution at the origin of the magneto-mechanical coupling with deformable bodies. An advantage of this approach is that the same mesh can be used by both methods during the resolution steps.
\end{abstract}

Index Terms - Finite-element method, integral method, magneto-mechanical coupling, nodal magnetic forces.

\section{INTRODUCTION}

$\mathbf{T}$ HE modeling of electromagnetic devices using deformable materials involves the resolution of the mechanical and electromagnetic equations including coupling terms in each physic. A classical method to solve numerically such problems is the finite-element method [1]. However, for devices with a huge volume of free space compared with the active structure or high size ratio between the geometric objects like micro-electro-mechanical systems (MEMS) [2], problems of accuracy could be present [3] for the resolution of the magnetostatic problem. To avoid this limitation, this paper investigates the magneto-mechanical coupling of devices composed of ferromagnetic and deformable materials in which the magnetic problem is solved with an integral formulation while the mechanical problem is solved by the finite-element method.

Considering only static case and no magnetostrictive effects, the equations of the magneto-mechanical problem can be solved separately. The magnetostatic problem is an open boundary problem for the magnetic field whereas the mechanical part is restricted to the solid domain. In this case, the integral formulations of the magnetostatic field problems are particularly advantageous for the numerical solution of open boundary problems which include magnetic materials. Indeed only the active regions containing these materials need to be discretized [4]. Therefore, a volume integral method is used to solve the magnetostatic problem.

With the assumptions of no magnetostrictive phenomena, only the magnetic forces are involved in the magnetomechanical coupling. A local application of the virtual work principle has been proposed in [5], to compute the nodal magnetic force distribution in the framework of the volume

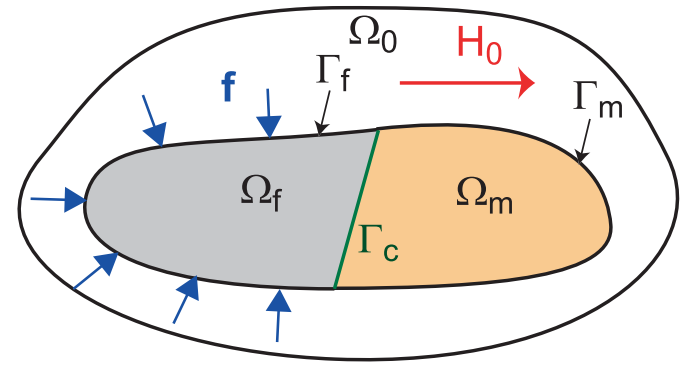

Fig. 1. Description of the magneto-mechanical problem.

integral method. This method is used here in order to compute a source term of a finite-element description of the mechanical problem. Advantage of this approach is to keep the same mesh for both resolutions and to consider only the active regions.

This paper presents in the first part a description of the problem with the associated equations. The second part is dedicated to the magneto-mechanical formulation in which a volume integral method is used to solve the magnetostatic problem. A local application of the virtual work principle, adapted to the framework of the integral methods, is proposed to compute the local magnetic forces. The finite-element method to solve the mechanical problem is also introduced. Finally, an application is given in the last part to illustrate the potential of the developed method.

\section{DESCRIPTION OF THE PROBLEM}

Let us consider the magneto-mechanical problem defined by the 2-D regions, $\Omega_{f}$ and $\Omega_{m}$, filled, respectively, with ferromagnetic and nonmagnetic materials (Fig. 1). Each of them has an isotropic linear elastic mechanical behavior. Both regions do not overlap and their union is noted $\Omega=\Omega_{f} \cup \Omega_{m}$. Let $\Gamma_{f}, \Gamma_{m}$, and $\Gamma$ be, respectively, the boundaries of the regions $\Omega_{f}, \Omega_{m}$, and $\Omega$. The regions $\Omega_{f}$ and $\Omega_{m}$ are in contact over the boundary $\Gamma_{c}=\Gamma_{f} \cap \Gamma_{m}$. The region $\Omega_{0}$ is associated to the free space. 
Considering only the static case, the magnetic behavior is governed by the Maxwell's equations

$$
\begin{aligned}
\nabla \times \mathbf{H} & =\mathbf{J} \\
\nabla \cdot \mathbf{B} & =0 .
\end{aligned}
$$

The magnetic soft behavior is considered linear. Therefore, in an isotropic homogeneous ferromagnetic material without magnetostrictive phenomena, the magnetic behavior law is defined by

$$
\mathbf{B}=\mu_{0}(\mathbf{H}+\mathbf{M})
$$

where $\mu_{0}$ is the magnetic permeability of free space and $\mathbf{M}$ the magnetization. The fields $\mathbf{M}$ and $\mathbf{H}$ are linked by

$$
\mathbf{M}=\chi \mathbf{H}
$$

where $\chi$ is the magnetic susceptibility.

The mechanical behavior is governed by the application of local equilibrium equations for a static case

$$
\nabla \cdot[\sigma]+\mathbf{f}=0
$$

where $[\sigma]$ is the stress tensor and $\mathbf{f}$ the volume force density. Only the magnetic interaction is considered (all other potential energy fields are neglected as well as the frictional forces), thus the volume force density is associated to the magnetic forces acting on the ferromagnetic material $\Omega_{f}$. The mechanical behavior law is defined by

$$
[\sigma]=\langle C\rangle[s]
$$

where $[s]$ is the strain tensor and $\langle C\rangle$ the stiffness tensor. The materials are considered homogeneous and isotropic with a elastic mechanical behavior. In this case, the stiffness tensor $\langle C\rangle$ can be expressed in function of Young's modulus $E$ and Poisson's ratio $v$

$$
C_{i j k l}=\frac{E}{1+v}\left(\frac{v}{1-2 v} \delta_{k l} \delta_{i j}+\frac{1}{2}\left(\delta_{i k} \delta_{j l}+\delta_{i l} \delta_{j k}\right)\right)
$$

where $\delta$ is the Kronecker's symbol. With the assumption of small deformations, the strain tensor is linked to the displacement field $\mathbf{u}$ (specifying the position of a point from a reference frame) by the linearized equation

$$
[s(\mathbf{u})]=\frac{1}{2}\left(\nabla \mathbf{u}+\nabla \mathbf{u}^{\mathrm{T}}\right) .
$$

\section{Magneto-Mechanical Formulation}

\section{A. Magnetostatic Formulation}

The magnetic field is written as the sum of the reduced magnetic field created by the magnetic material $\mathbf{H}_{\text {red }}$ and the magnetic source field created by currents flows $\mathbf{H}_{0}$

$$
\mathbf{H}=\mathbf{H}_{\mathrm{red}}+\mathbf{H}_{0} .
$$

The Maxwell's equations (1) and (2) are satisfied when the reduced magnetic field $\mathbf{H}_{\text {red }}$ is expressed at any point of coordinates $\mathbf{r}$ by [6]

$$
\mathbf{H}_{\mathrm{red}}(\mathbf{M}, \mathbf{r})=-\frac{1}{4 \pi} \nabla\left(\int_{\Omega_{a}} \frac{\mathbf{M}\left(\mathbf{r}^{\prime}\right) \cdot\left(\mathbf{r}-\mathbf{r}^{\prime}\right)}{\left|\mathbf{r}-\mathbf{r}^{\prime}\right|^{3}} \mathrm{~d} \Omega^{\prime}\right) .
$$

The magnetic field $\mathbf{H}$ (11), solution of the magnetostatic problem, verifies the (10) and (4) at any point of the ferromagnetic region $\Omega_{f}$

$$
\mathbf{H}(\mathbf{r})=\mathbf{H}_{\mathrm{red}}(\chi \mathbf{H}, \mathbf{r})+\mathbf{H}_{0}(\mathbf{r}) .
$$

The simply connected region $\Omega_{f}$ containing no current sources, the magnetic fields $\mathbf{H}$ and $\mathbf{H}_{0}$ derive, respectively, from the scalar potentials $\Phi$ and $\Phi_{0}$. The integration of (11) leads to the following volume integral equation [7], [8]:

$$
\Phi(\mathbf{r})+\frac{1}{4 \pi} \int_{\Omega_{f}} \chi \frac{\nabla \Phi\left(\mathbf{r}^{\prime}\right) \cdot\left(\mathbf{r}-\mathbf{r}^{\prime}\right)}{\left|\mathbf{r}-\mathbf{r}^{\prime}\right|^{3}} \mathrm{~d} \boldsymbol{\Omega}^{\prime}=\Phi_{0} .
$$

The region $\Omega_{f}$ is meshed with tetrahedra elements using first order shape functions

$$
\Phi=\sum_{i} \alpha_{i} \Phi_{i}
$$

where $\alpha_{i}$ and $\Phi_{i}$ are, respectively, the shape function and the degree of freedom of the potential $\Phi$ associated to the $i$ th node of the mesh. A collocation method at mesh nodes is used to solve (12) and leads to solve the following system of algebraic equations

$$
([I]+[A(\chi)]) \Phi=\Phi_{0}
$$

with

$$
\begin{aligned}
I_{i j} & =\delta_{i j} \\
A(\chi)_{i j} & =\frac{1}{4 \pi} \int_{\Omega_{f}} \chi \frac{\nabla \alpha_{j}\left(\mathbf{r}^{\prime}\right) \cdot\left(\mathbf{r}_{i}-\mathbf{r}^{\prime}\right)}{\left|\mathbf{r}_{i}-\mathbf{r}^{\prime}\right|^{3}} \mathrm{~d} \Omega^{\prime}
\end{aligned}
$$

where $\mathbf{r}_{i}$ is the coordinates of the $i$ th node. There are no direct analytical expressions for the source potential $\Phi_{0}$. A numerical approach presented in [9] is then used.

The volume integral method allows to mesh only the active materials. The main drawback is that the interaction matrix $[A]$ is full. The compression methods can overcome this limitation [10].

\section{B. Local Magnetic Force Computation}

Several methods are available in the literature to compute the local magnetic forces: Maxwell's tensor, virtual works, equivalent source or charge methods, eggshell method [11] etc. These methods are several times compared each other [12]. The virtual work principle is more general [13] and has already been successfully applied to compute magnetic forces inside electro-magnetic MEMS [1]. Considering that, an adaptation of the virtual work principle in the framework of the volume integral method is used [5].

A virtual displacement $\mathbf{u}^{n}$ is applied on the mesh node $n$. According to the virtual work principle [14], the component along $\mathbf{u}^{n}$ of the nodal magnetic force $F_{u}^{n}$ is deduced from

$$
F_{u}^{n}=\left.\frac{\mathrm{d} \omega_{\mathrm{mag}}^{\mathrm{co}}}{\mathrm{d} \mathbf{u}^{n}}\right|_{\mathrm{I}}
$$

where $I$ is the electric current which is kept constant and $\omega_{\mathrm{mag}}^{\mathrm{co}}$ the magnetic co-energy. This last could be written [15]

$$
\omega_{\mathrm{mag}}^{\mathrm{co}}=\omega_{\mathrm{ferro}}^{\mathrm{co}}+\frac{\mu_{0}}{2} \int_{\Omega \cup \Omega_{0}} \mathbf{H}_{0} \cdot \mathbf{H}_{0} \mathrm{~d} \Omega
$$


with

$$
\omega_{\text {ferro }}^{\text {co }}=\frac{\mu-\mu_{0}}{2} \int_{\Omega_{f}} \mathbf{H} \cdot \mathbf{H}_{0} \mathrm{~d} \Omega .
$$

The expression (18) is used in (17) to obtain

$$
F_{u}^{n}=\left.\frac{\mathrm{d} \omega_{\text {ferro }}^{\text {co }}}{\mathrm{d \mathbf {u } ^ { n }}}\right|_{\mathrm{I}}
$$

The relation (20) is well suited for the integral formulations, due to the fact that the domain of the integral (19) is limited to the ferromagnetic region.

Using the change of basis between the physical mesh element $\Omega_{e}$ and the reference element $\hat{\Omega}$ described by the Jacobian matrix of the transformation $\left[J_{e}\right]$, the expression (20) can be developed into

$$
\begin{aligned}
F_{u}^{n}=\sum_{e} \frac{\mu-\mu_{0}}{2} \int_{\hat{\Omega}} & {\left[\left(\mathbf{H} \cdot \frac{\mathrm{d} \mathbf{H}_{0}}{\mathrm{~d} \mathbf{u}^{n}}+\frac{\mathrm{d} \mathbf{H}}{\mathrm{d} \mathbf{u}^{n}} \cdot \mathbf{H}_{0}\right)\left|J_{e}\right|\right.} \\
& \left.+\left(\mathbf{H} \cdot \mathbf{H}_{0}\right) \frac{\mathrm{d}\left|J_{e}\right|}{\mathrm{d} \mathbf{u}^{n}}\right] \mathrm{d} \Omega .
\end{aligned}
$$

The computation of each derivative term has been discussed in [5].

Each component of the nodal magnetic force $F^{n}$ is obtained taking the virtual displacement $\mathbf{u}^{n}$, respectively, as the basis vectors ( $\mathbf{e}_{1}, \mathbf{e}_{2}$, and $\left.\mathbf{e}_{3}\right)$. Therefore, using the relation (21) the nodal magnetic force $\mathbf{F}^{n}$ is given by

$$
\mathbf{F}^{n}=\sum_{k=1}^{3} F_{e_{k}}^{n} \mathbf{e}_{k}
$$

This adaptation of the virtual work principle allows computing local magnetic forces without computation in the free space. Its main drawback is the resolution of two systems of algebraic equations for each mesh node and each space dimension.

\section{Mechanical Formulation}

Let us consider the region $\Omega$ is submitted to mechanical displacement on boundary $\Gamma^{u}$ and to applied mechanical stresses on boundary $\Gamma^{\sigma}$. The corresponding boundary conditions are defined by

$$
\begin{aligned}
& {[\sigma] \cdot \mathbf{n}=\mathbf{f}^{\sigma} \text { on } \Gamma^{\sigma}} \\
& \mathbf{u}=\mathbf{u}_{0} \text { on } \Gamma^{u}
\end{aligned}
$$

where $\Gamma^{\sigma}$ and $\Gamma^{u}$ are the parts of the boundary $\Gamma$ (Fig. 1).

The weak formulation associated to the mechanical problem (5) is defined by

$$
\int_{\Omega} \mathbf{w} \nabla \cdot[\sigma] \mathrm{d} \Omega=-\int_{\Omega} \mathbf{w} \cdot \mathbf{f} \mathrm{d} \Omega \quad \forall \mathbf{w}
$$

where $\mathbf{w}$ is a test function verifying the boundary condition (23). Using the rule product for the divergence, the Stoke's theorem and the boundary conditions (23) and (24), the weak formulation (25) can be written

$$
\int_{\Omega}[s(\mathbf{w})][\sigma] \mathrm{d} \Omega=\int_{\Omega} \mathbf{w} \cdot \mathbf{f} \mathrm{d} \Omega+\int_{\Gamma^{\sigma}} \mathbf{w} \cdot \mathbf{f}^{\sigma} \mathrm{d} \Gamma, \quad \forall \mathbf{w} .
$$

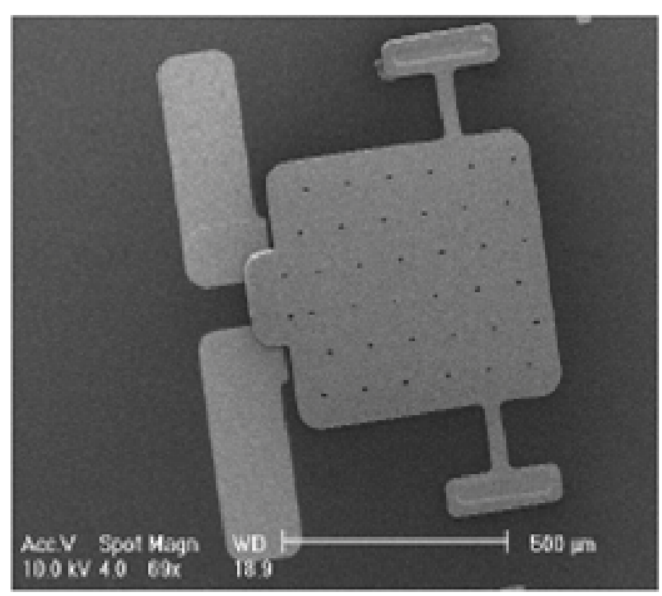

Fig. 2. Description of the MEMS.

The region $\Omega_{m}$ is meshed with tetrahedra elements using first order shape functions

$$
\mathbf{u}=\sum_{i} \alpha_{i} \mathbf{U}_{i}
$$

where $\alpha_{i}$ and $\mathbf{U}_{i}$ are, respectively, the shape function and the vector degree of freedom of the displacement $\mathbf{u}$ associated to the $i$ th node of the mesh.

The finite-element method applied to the weak mechanical formulation (26) leads to the resolution of the algebraic equation system

$$
[K] \mathbf{U}=\mathbf{F}+\mathbf{Q}
$$

with

$$
\begin{aligned}
K_{i j} & =\int_{\Omega}\left[s\left(\lambda_{\mathbf{j}}\right)\right]\langle C\rangle\left[s\left(\lambda_{\mathbf{i}}\right)\right] \mathrm{d} \Omega \\
F_{i} & =\int_{\Omega} \lambda_{\mathbf{i}} \cdot \mathbf{f} \mathrm{d} \Omega \\
Q_{i} & =\int_{\Gamma} \lambda_{\mathbf{i}} \cdot \mathbf{f}^{\sigma} \mathrm{d} \Gamma
\end{aligned}
$$

where $\lambda_{i}$ is the vector shape function associated to the vector degree of freedom $i$. The vector $\mathbf{F}$ is equal to the vector of the nodal magnetic force (22).

\section{ApPlicAtion}

Let us study, a MEMS actuator (Fig. 2) [16] in which an iron plate is fixed to a beam which is embedded at its extremities $\left[\mathbf{u}_{0}=\mathbf{0}\right.$ for the boundary condition (24)]. No external force is applied to the structure $\left[\mathbf{f}^{\sigma}=\mathbf{0}\right.$ for the boundary condition (23)]. The description of the MEMS and the corresponding mesh are presented on the Fig. 3. The magnetic behavior of the MEMS is considered linear with an isotropic homogeneous ferromagnetic material of magnetic permeability $1000(\mathrm{H} / \mathrm{m})$. Its mechanical behavior is considered elastic with a homogeneous and isotropic material of Young's modulus $E=110 \mathrm{GPa}$ and Poisson's ratio $v=0.3$. The MEMS is actuated by a magnetic field produced by a source. Without magnetostrictive effects, the magnetic forces are the origin of 


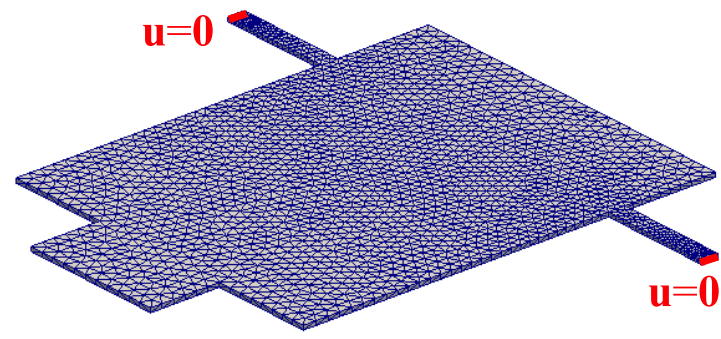

Fig. 3. Model of the MEMS with the boundary conditions.

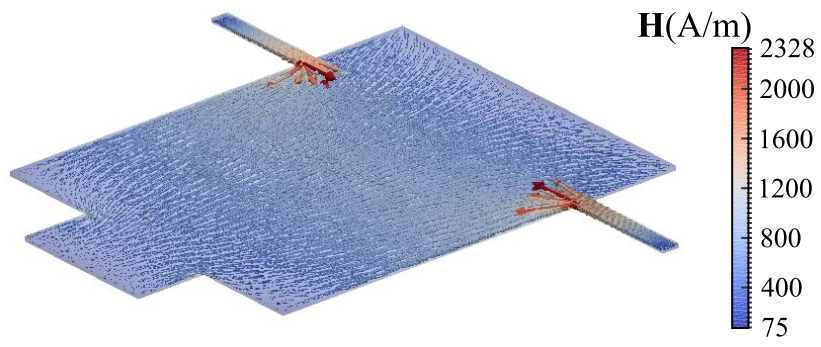

Fig. 4. Magnetic field distribution.

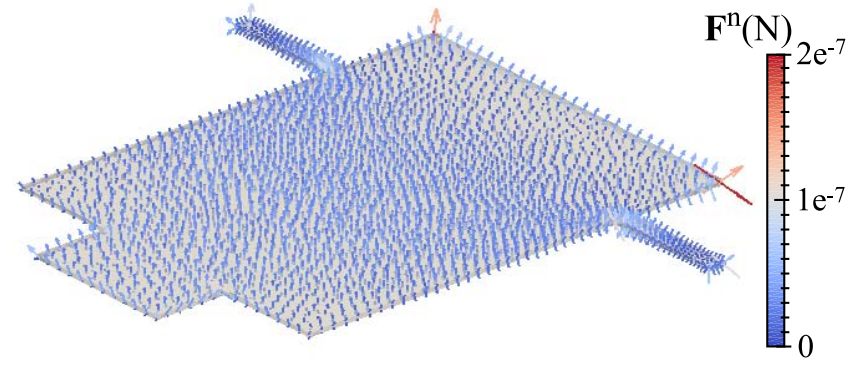

Fig. 5. Nodal magnetic forces distribution.

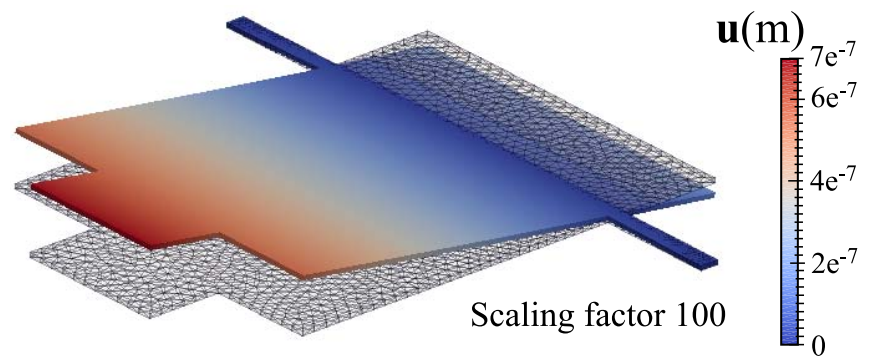

Fig. 6. Displacement distribution with the new position of the MEMS.

the magneto-mechanical coupling and generate torsional strain of the beam which rotates around its axis.

The previous volume integral method (14) is used to solve the magnetostatic problem. The computed magnetic field is given in Fig. 4. The virtual work method (22) is applied to compute the magnetic nodal force distribution which is presented on Fig. 5. These nodal forces become the source term of the mechanical problem which is solved using the finite-element method (28). Fig. 6 represents the computed displacement with the new position of the MEMS and the reference configuration. This is in agreement with the torsion motion of the beam which rotates around its axis.

\section{CONClusion}

A magneto-mechanical coupling problem is solved in the framework of the volume integral and finite-element methods. The resolution of the magnetic behavior with the volume integral method allows to compute efficiently the nodal magnetic force distribution by an adapted virtual work principle formulation. This force distribution is then a source term for the mechanical problem which is achieved by finite-element method. Associated to an accurate magnetic force computation, the advantages of this approach is to consider only the active regions during the magneto-mechanical resolution, and to use the same mesh during the resolution steps. A work in progress is the investigation of the nonlinear case.

\section{REFERENCES}

[1] N. Galopin, X. Mininger, F. Bouillault, and L. Daniel, "Finite element modeling of magnetoelectric sensors," IEEE Trans. Magn., vol. 44, no. 6, pp. 834-837, Jun. 2008.

[2] O. Cugat, J. Delamare, and G. Reyne, "Magnetic micro-actuators and systems (MAGMAS)," IEEE Trans. Magn., vol. 39, no. 5, pp. 3607-3612, Nov. 2003.

[3] H. L. Rakotoarison, V. Ardon, O. Chadebec, B. Delinchant, S. Guerin, and J. L. Coulomb, "Formal sensitivity computation of magnetic moment method," IEEE Trans. Magn., vol. 44, no. 6, pp. 1014-1017, Jun. 2008.

[4] B. Trowbridge, "Integral equations in electromagnetics," Int. J. Numer Model., Electron. Netw., Devices Fields, vol. 9, nos. 1-2, pp. 3-17, 1996.

[5] A. Carpentier, N. Galopin, O. Chadebec, G. Meunier, and C. Guérin, "Application of the virtual work principle to compute magnetic forces with a volume integral method," Int. J. Numer. Model., to be published.

[6] E. Durand, Magnétostatique. Paris, France: Masson, 1968.

[7] L. Han, L.-S. Tong, and J. Yang, "Integral equation method using total scalar potential for the simulation of linear or nonlinear 3D magnetostatic field with open boundary," IEEE Trans. Magn., vol. 30, no. 5, pp. 2897-2900, Sep. 1994.

[8] L. Kettunen, K. Forsman, D. Levine, and W. Gropp, "Volume integral equations in non-linear 3-D magnetostatics," Int. J. Numer. Methods Eng., vol. 38, no. 16, pp. 2655-2675, 1995.

[9] A. Carpentier, O. Chadebec, N. Galopin, G. Meunier, and B. Bannwarth, "Resolution of nonlinear magnetostatic problems with a volume integral method using the magnetic scalar potential," IEEE Trans. Magn., vol. 49, no. 5, pp. 1685-1688, May 2013.

[10] J. Carrier, L. Greengard, and V. Rokhlin, "A fast adaptive multipole algorithm for particle simulations." SIAM J. Sci. Stat. Comput., vol. 9, no. 4, pp. 669-686, 1988.

[11] F. Henrotte, G. Deliège, and K. Hameyer, "The eggsheel approach for the computation of the electromagnetic forces in 2D and 3D," Int. J. Comput. Math. Electr. Electron. Eng., vol. 23, no. 4, pp. 996-1005, 2004.

[12] L. Vandevelde and J. A. A. Melkebeek, "A survey of magnetic force distributions based on different magnetization models and on the virtual work principle," IEEE Trans. Magn., vol. 37, no. 5, pp. 3405-3409, Sep. 2001.

[13] A. Bossavit, "Virtual power principle and Maxwell's tensor: Which comes first?" Int. J. Comput. Math. Electr. Electron. Eng., vol. 30, no. 6 , pp. 1804-1814, 2011.

[14] Z. Ren and A. Razek, "Local force computation in deformable bodies using edge elements,' IEEE Trans. Magn., vol. 28, no. 2, pp. 1212-1215, Mar. 1992.

[15] J. A. Stratton, Electromagnetic Theory. New York, NY, USA: McGrawHill, 1941.

[16] Schneider Electric, "Microsystème à commande électromagnétique," EP Patent 1836714, Sep. 26, 2007. 